\title{
Defect-rich GaN interlayer facilitating the annihilation of threading dislocations in polar GaN crystals grown on (0001)-oriented sapphire substrates
}

Cite as: J. Appl. Phys. 126, 085301 (2019); https://doi.org/10.1063/1.5092284

Submitted: 11 February 2019 . Accepted: 06 August 2019. Published Online: 23 August 2019

M. Barchuk (D, M. Motylenko, T. Schneider, M. Förste, C. Röder, A. Davydok, S. Lazarev (D), C. Schimpf, C. Wüstefeld, O. Pätzold, and D. Rafaja (i)
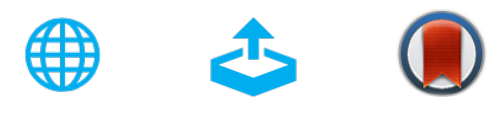

View Online

Export Citation

CrossMark

\section{ARTICLES YOU MAY BE INTERESTED IN}

Extra half-plane shortening of dislocations as an origin of tensile strain in Si-doped (Al)GaN Journal of Applied Physics 126, 085701 (2019); https://doi.org/10.1063/1.5111664

Formation of nitrogen-vacancy centers in $4 \mathrm{H}-\mathrm{SiC}$ and their near infrared photoluminescence properties

Journal of Applied Physics 126, 083105 (2019); https://doi.org/10.1063/1.5099327

The role of Mg bulk hyper-doping and delta-doping in low-resistance GaN homojunction tunnel diodes with negative differential resistance

Journal of Applied Physics 126, 083110 (2019); https://doi.org/10.1063/1.5112498

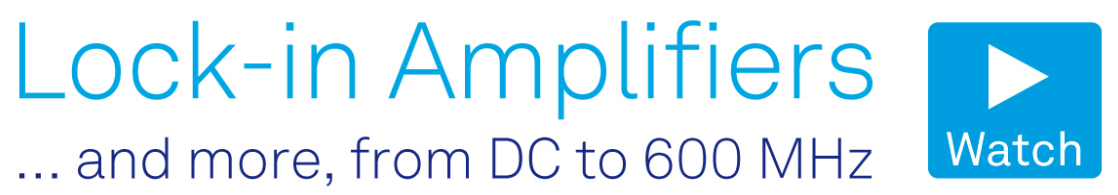




\title{
Defect-rich GaN interlayer facilitating the annihilation of threading dislocations in polar GaN crystals grown on (0001)-oriented sapphire substrates
}

Cite as: J. Appl. Phys. 126, 085301 (2019); doi: 10.1063/1.5092284

Submitted: 11 February 2019 - Accepted: 6 August 2019 .

Published Online: 23 August 2019

M. Barchuk, ${ }^{7}$ (D) M. Motylenko, ${ }^{7}$ T. Schneider, $^{2}$ M. Förste, ${ }^{2}$ C. Röder, ${ }^{3}$ A. Davydok, ${ }^{4}$ S. Lazarev, ${ }^{5,6}$ (D) C. Schimpf, ${ }^{1}$ C. Wüstefeld, ${ }^{1}$ O. Pätzold, ${ }^{2}$ and D. Rafaja ${ }^{1, a)}$ (D)

\author{
AFFILIATIONS \\ ${ }^{1}$ Institute of Materials Science, TU Bergakademie Freiberg, Gustav-Zeuner-Str. 5, D-09596 Freiberg, Germany \\ ${ }^{2}$ Institute of Nonferrous Metallurgy and Purest Materials, TU Bergakademie Freiberg, Leipziger Str. 34, D-09596 Freiberg, Germany \\ ${ }^{3}$ Institute of Theoretical Physics, TU Bergakademie Freiberg, Leipziger Str. 23, D-09596 Freiberg, Germany \\ ${ }^{4}$ Institute of Materials Research, Helmholtz-Zentrum Geesthacht, Notkestr. 85, D-22607 Hamburg, Germany \\ ${ }^{5}$ Deutsches Elektronen-Synchrotron DESY, Notkestr. 85, D-22607 Hamburg, Germany \\ ${ }^{6}$ National Research Tomsk Polytechnic University (TPU), Lenin Avenue 30, 634050 Tomsk, Russian Federation
}

a) Author to whom correspondence should be addressed: rafaja@ww.tu-freiberg.de

\begin{abstract}
The interaction of microstructure defects is regarded as a possible tool for the reduction of the defect density and improvement of the crystal quality. In this study, this general approach is applied to reduce the density of threading dislocations in GaN crystals grown using high-temperature vapor phase epitaxy directly on (0001)-oriented sapphire substrates. The GaN crystals under study were deposited in three steps with different process temperatures, growth rates, and ammonia flows. The first GaN layer accommodates the lattice misfit between sapphire and gallium nitride. Thus, it contains a high number of randomly distributed threading dislocations. The next GaN layer, which is internally structured and defect-rich, bends and bunches these dislocations and facilitates their annihilation. The uppermost GaN layer mainly contains bunched threading dislocations terminating large areas of almost defect-free GaN. In order to be able to visualize and to quantify the microstructure changes in individual parts of the sandwich-like structure, the samples were investigated using nanofocused synchrotron diffraction, confocal micro-Raman spectroscopy, and transmission electron microscopy. The transmission electron microscopy provided information about the kind of microstructure defects and their mutual interaction. The synchrotron diffraction and the micro-Raman spectroscopy revealed the depth profiles of dislocation density and lattice parameters.
\end{abstract}

Published under license by AIP Publishing. https://doi.org/10.1063/1.5092284

\section{INTRODUCTION}

Gallium nitride $(\mathrm{GaN})$ is a wide bandgap semiconductor which is used in many electronic and optoelectronic applications. $\mathrm{GaN}$ crystals are typically produced in the form of thick layers deposited on foreign substrates such as $\mathrm{Al}_{2} \mathrm{O}_{3}, \mathrm{SiC}, \mathrm{Si}$, etc. ${ }^{1,2}$ The quality of the GaN layers and their optical and optoelectronic properties depend strongly on the kind and density of microstructure defects. The initial defect structure is affected by the choice of the substrate and its roughness, by the crystallographic orientations of the GaN layer and the respective substrate, and by the parameters of the deposition process.

The main type of the microstructure defects in polar, i.e., (0001)-oriented GaN layers grown on foreign substrates, are threading dislocations (TDs) to which dislocation lines are aligned along the crystallographic direction [001]. ${ }^{3,6-8}$ Although in the microstructure models used for the determination of the dislocation density, ${ }^{9-12}$ the TDs are assumed to propagate mainly in the 
vertical direction [001], and they tend to bend, bunch, or annihilate, in particular, if they interact with other crystal structure defects. $^{13-16}$ Consequently, the interaction of TDs with some microstructure defects leads to an effective reduction of the TD density in GaN epilayers.

Based on the interaction of microstructure defects with certain microstructure features, several approaches were developed that improve the quality of $\mathrm{GaN}$ layers by deflecting threading dislocations from the [001] direction. Bourret-Courchesne et al. ${ }^{17}$ have shown that the TD density in GaN layers can be reduced by a GaN interlayer deposited at an intermediate temperature. This interlayer forces the TDs to bend in the vicinity of the interface between the interlayer and the underlying $\mathrm{GaN}$ and consequently helps to reduce their density. Other approaches reducing the TD density are the deposition of a $\mathrm{SiN}_{\mathrm{x}}$ mask that terminates the propagation of TDs into the upper GaN layer ${ }^{18,19}$ and the use of AlN nucleation layers on (0001)-oriented sapphire substrates that modifies the lattice mismatch between the substrate and $\mathrm{GaN}^{20-22}$ In a recent publication, Stanchu et al. described the positive effect of compositionally graded $\mathrm{AlGaN}$ buried layers in GaN/AlGaN/GaN stacks on the lattice strain relaxation and on the annihilation of TDs. ${ }^{23}$ Among the nowadays actively developed techniques which support bending and bunching of the TDs in GaN, the selective area growth (SAG), ${ }^{24-28}$ the epitaxial lateral overgrowth (ELO), ${ }^{29-33}$ and the facet-controlled epitaxial lateral overgrowth (FACELO) ${ }^{34-36}$ are to be mentioned.

In this work, we investigate GaN stacks containing a defect-rich GaN interlayer, which were grown by modified hightemperature vapor phase epitaxy (HTVPE) directly on (0001)-oriented sapphire substrates. ${ }^{37}$ The HTVPE technique offers high flexibility of the deposition process through the variation of the flow of precursors, the source and substrate temperatures, the reactor pressure, and the growth rate. ${ }^{38-40}$ To reduce the TD density, we deposited a defect-rich GaN interlayer in which the TDs interact with other microstructure defects. This concept of the defect engineering employs a variation of the deposition parameters that results in the emergence of (partial) stacking faults (SFs), ${ }^{40,41}$ voids, ${ }^{41}$ and occasionally inverse domain boundaries. ${ }^{42}$ These intentionally created microstructural defects change the atomic arrangement in the vicinity of $\mathrm{TDs}^{31,43}$ and hinder the propagation of TDs toward the surface of the GaN crystals. Consequently, the microstructure defects in the intermediate layer decrease the TD density in the uppermost GaN layer.

The microstructure defects induced by the change of the deposition parameters and their interaction with TDs were investigated using nanofocused synchrotron diffraction in transmission mode, transmission electron microscopy (TEM), selected area electron diffraction, and micro-Raman spectroscopy. These methods revealed the depth dependence of the kind and density of microstructure defects and the depth profile of the lattice parameters. The synchrotron, TEM, and micro-Raman experiments were complemented by laboratory high-resolution X-ray diffraction (HRXRD) performed in coplanar reflection mode. The results of these microstructure studies contributed to the understanding of the mechanisms behind the interaction between different microstructure defects and allow us to apply this knowledge for the improvement of the crystal quality.

\section{EXPERIMENTAL}

\section{A. Growth of the GaN stacks}

The GaN samples under study were grown by HTVPE in a vertical reactor $^{37}$ directly on (0001)-oriented sapphire substrates having a size of $15 \times 15 \mathrm{~mm}^{2}$. A complete sample on a sapphire substrate ( $\mathrm{S} 1$ in Fig. 1) consists of a stack of a plane template, a structured intermediate layer, and a plane top layer. The deposition of a template layer started from a recrystallized nucleation layer. For template growth, the temperatures of the substrate and the Ga source were set to $1100^{\circ} \mathrm{C}$ and $1250^{\circ} \mathrm{C}$, respectively. The total gas flow in the reactor was 3.0 standard liter per minute $(\mathrm{slm})$ with an ammonia flow of $0.3 \mathrm{slm}$ and a hydrogen flow of $0.6 \mathrm{slm}$. Further constituents of the working gas were $\mathrm{Ar}$ and $\mathrm{N}_{2}$. The total pressure was $985 \mathrm{mbar}$ and the resulting growth rate was $2.5 \mu \mathrm{m} / \mathrm{h}$.

The structured layer was deposited under changed process conditions in order to reduce the surface diffusivity of the species. The substrate temperature was $1000^{\circ} \mathrm{C}$, the source temperature $1300{ }^{\circ} \mathrm{C}$, the total gas flow $3.0 \mathrm{slm}$, the $\mathrm{NH}_{3}$ flow $0.5 \mathrm{slm}$, the $\mathrm{H}_{2}$ flow $0.9 \mathrm{slm}$, the total pressure $55 \mathrm{mbar}$, and the growth rate $133 \mu \mathrm{m} / \mathrm{h}$. The top layer was deposited with the same parameters like the template with the exception of the $\mathrm{NH}_{3}$ flow, which was increased to $1 \mathrm{slm}$. The different process parameters lead to significant differences in the $[\mathrm{N}] /[\mathrm{Ga}](\mathrm{V} / \mathrm{III})$ ratio during the growth, which was about 1250 for the template, 40 for the structured layer, and 5000 for the top layer. These V/III ratios were estimated from the results of numerical simulation of the gas phase transport in the HTVPE reactor using Comsol Multiphysics as described by Lukin et al. ${ }^{39}$

In order to make the individual deposition steps accessible for microstructure analysis using complementary techniques, three samples with different architectures were produced (Fig. 1). Sample S3 comprises just the nucleation layer and the GaN template, sample S2 contains the structured GaN layer in addition to the components of sample S3, and sample S1 consists of the whole layer stack.

\section{B. TEM analyses}

The TEM analyses were carried out in a transmission electron microscope JEM-2200FS from JEOL, which is equipped with a field emission gun (FEG), a Cs corrector located in the illumination system, a high-resolution objective lens $(\mathrm{Cs}=0.5 \mathrm{~mm})$, and an
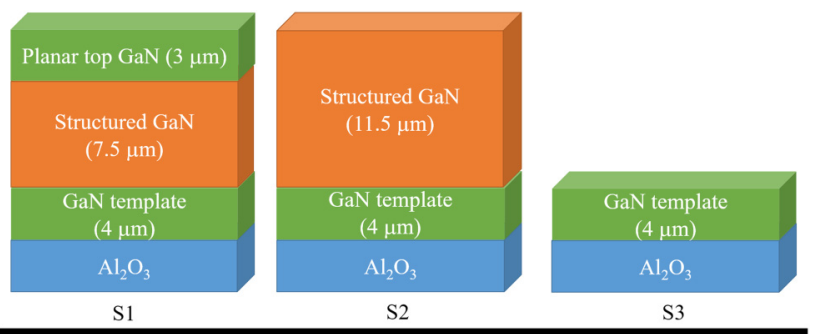

FIG. 1. Schematic structure of the samples under study. 
$\Omega$-filter. The microscope was operated in scanning mode (STEM) at the acceleration voltage of $200 \mathrm{kV}$. Bright field (BF) and dark field (DF) STEM images were collected by a bright field and a high angle annular dark field detector, respectively. For acquisition of the selected area electron diffraction (SAED) patterns, a highly sensitive $2 \mathrm{~K} \times 2 \mathrm{~K}$ charge-coupled device (CCD) camera was employed. The STEM micrographs and the SAED patterns were analyzed using the Digital Micrograph software from Gatan. The TEM analyses were done on a [100]-oriented cross-section of sample S1 that was prepared using Precision Ion Polishing System (PIPS II) from Gatan. The thickness of the specimen was between 150 and $300 \mathrm{~nm}$.

\section{Raman spectroscopy}

Micro-Raman spectroscopic measurements were carried out in order to monitor the residual stress within the GaN sublayers of the investigated samples. The measurements were performed using a Labram HR 800 Horiba Jobin Yvon spectrometer (Villeneuve d'Ascq, France) at room temperature in backscattering geometry. The Raman spectra were excited using the $532 \mathrm{~nm}(2.33 \mathrm{eV})$ line of a frequency-doubled Nd:YAG laser. By means of the confocal technique, Raman spectra were recorded at a desired depth below the sample's surface by moving the focal plane of the laser optics. The vertical resolution of the micro-Raman spectroscopic measurements was about $2.5 \mu \mathrm{m}$, and the lateral resolution was about $1 \mu \mathrm{m} .{ }^{44}$ The residual stress $(\sigma)$ was calculated from the shift of the nonpolar $E_{2}$ (high) Raman mode from the spectral position $\omega_{E_{2}(\text { high })}^{0}=567.6 \mathrm{~cm}^{-1}$ that corresponds to the unstrained $\mathrm{GaN},{ }^{45,46}$

$$
\sigma=\left(\omega_{E_{2}(h i g h)}^{\text {meas }}-\omega_{E_{2}(h i g h)}^{0}\right) / K_{E_{2}(h i g h)} .
$$

Assuming a biaxial stress in the $c$-plane, the linear stress coefficient $K_{E_{2}(\text { high })}=-(2.7 \pm 0.3) \mathrm{cm}^{-1} \mathrm{GPa}^{-1}$ was used. In order to analyze the positions of the spectral lines as precise as possible, the frequency and background were corrected, and the $E_{2}$ (high) mode was fitted by a Lorentzian function.

\section{X-ray diffraction experiment at synchrotron}

The depth-resolved synchrotron diffraction experiment was performed on a cross-section of sample S1 in transmission mode at the P03 nanofocus end-station at PETRA III (DESY, Hamburg, Germany). ${ }^{47}$ This end-station is located $97 \mathrm{~m}$ downstream the undulator. The wavelength $\lambda=0.95591 \AA$ was selected by a (111)-oriented silicon crystal monochromator having a spectral resolution of $\Delta \lambda / \lambda=2 \times 10^{-4}$. The corresponding energy of $13 \mathrm{keV}$ was chosen to avoid the absorption edge of $\mathrm{Ga}$ at $10.37 \mathrm{keV}$. The primary X-ray beam was focused on the sample employing the Kirkpatrick Baez mirrors from JTEC. The cross-section of the beam was about $300 \times 300 \mathrm{~nm}^{2}$, its divergence $1 \times 1.3 \mathrm{mrad}^{2} .48$ The diffracted signal was recorded using a high-resolution CCD camera from Photonic Science with $2940 \times 2940$ pixels and the pixel size of $61 \times 61 \mu \mathrm{m}^{2}$. The detector was placed approximately $83 \mathrm{~cm}$ downstream the sample to reach a desired resolution in a reciprocal space of $5 \times 10^{-4} \AA^{-1}$ and to fulfill the far-field conditions of X-ray diffraction. ${ }^{49}$
For the synchrotron experiment, a cross-section of sample S1 was prepared and thinned down to $80 \mu \mathrm{m}$ by mechanical polishing. At this sample thickness, approximately $0.7 \%$ of the primary X-ray beam intensity is still transmitted as calculated for the linear absorption coefficient of $625.16 \mathrm{~cm}^{-1}$. ${ }^{49}$ The sample was mounted in a goniometer in such a way that the original sample surface was aligned vertically and parallel to the incoming synchrotron beam as shown in Fig. 2. During the synchrotron measurements, a twodimensional cut through the $10 \overline{1} 0 \mathrm{GaN}$ reciprocal lattice point was recorded by the CCD detector for each selected position of the primary beam on the cross-section of the sample (denoted by $r$ in Fig. 2). Individual frames were recorded by moving the sample in the horizontal direction with a step size of $1 \mu \mathrm{m}$. The lateral homogeneity of the sample was inspected by carrying out the horizontal scans at different vertical positions of the sample. The exposition time was $30 \mathrm{~s}$ per frame. Assuming the resolution in a reciprocal space of $5 \times 10^{-4} \AA^{-1}$ (s. above), the angular resolution of the detector is $16 \operatorname{arcsec}(2 \theta)$, when the Bragg peak $10 \overline{1} 0$ of $\mathrm{GaN}$ is measured. Broadening of the diffraction spot in the vertical direction caused by the sample thickness of $80 \mu \mathrm{m}$ was estimated to be approximately $30 \mu \mathrm{m}$, which is a half of the detector pixel size and, therefore, can be neglected.

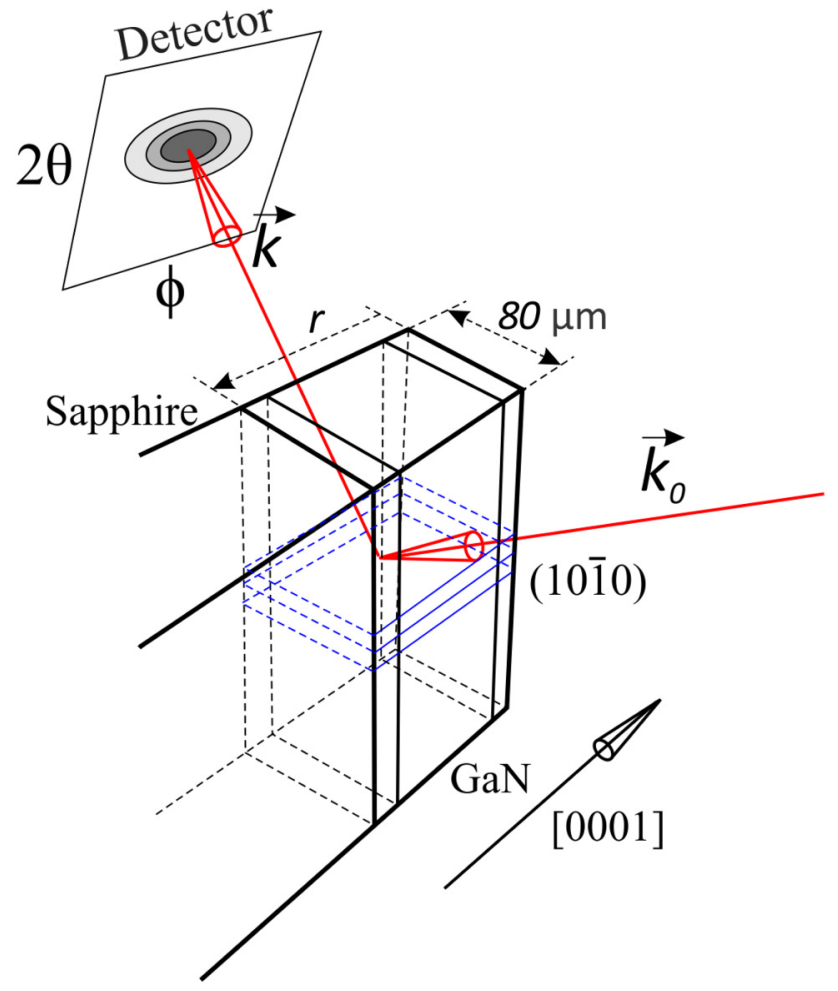

FIG. 2. Scheme of the synchrotron diffraction experiment performed in transmission mode on the $\mathrm{GaN}$ lattice planes (1010). The growth direction [001] is oriented horizontally. $\vec{k}_{0}$ and $\vec{k}$ are the wave vectors of the primary and diffracted beam, respectively. 


\section{E. Laboratory X-ray diffraction experiments}

Laboratory X-ray diffraction (XRD) measurements were carried out in the coplanar reflection geometry and comprised reciprocal space mapping of the GaN reflections 0004 and $11 \overline{2} 4$. For the XRD experiments, a triple-axis diffractometer from Seifert/ FPM was employed that was equipped with a $\mathrm{Cu}$ anode, a Eulerian cradle, and a scintillation detector. The primary X-ray beam was monochromatized by a (111)-oriented silicon single crystal to the wavelength $\lambda_{\mathrm{CuK} \alpha_{1}}=1.54056 \AA$. Another (111)-oriented Si single crystal was used as an analyzer in the diffracted X-ray beam. ${ }^{41}$ The reciprocal space mapping was performed as a series of radial $(\omega-2 \theta)$ scans at different sample inclinations $(\omega)$. The instrumental line broadening of coplanar $\mathrm{GaN}$ reflections was estimated to be below 16 arcsec. ${ }^{16}$

\section{RESULTS AND DISCUSSION}

\section{A. Microstructure changes across the GaN stack}

The transmission electron micrographs recorded in the dark-field (DF) STEM mode (Fig. 3) show the microstructure changes that were induced by the growth of the GaN layer stack under the above mentioned conditions. The template layer contains mainly TDs, which appear in Fig. 3(a) as bright lines along the crystallographic direction [001]. The DF STEM imaging cannot distinguish between screw and edge TDs. Thus, only the total density of TDs of about $10^{10} \mathrm{~cm}^{-2}$ could be estimated, which corresponds to the mean dislocation distances of approximately $0.1 \mu \mathrm{m}$. In the template, the TDs are more or less equidistantly distributed. Still, an onset of the dislocation bunching is visible. The beginning of the structured layer [red line in Fig. 3(a)] is dominated by freestanding columns and voids. These features make the interface between the template and the structured layer uneven-in addition to the initial roughness induced by the template layer.
The central part of the structured layer consists of vertical GaN columns, which are separated from each other by dislocation walls and occasional voids [Fig. 3(b)]. In the DF STEM micrograph, the dislocation walls appear bright and the voids dark. The average lateral size of the columns is approximately $1 \mu \mathrm{m}$. In most cases, the columns grow through the entire structured layer. Nevertheless, they contain SFs on basal planes, which appear in Fig. 3(b) as bright lines that are perpendicular to the growth direction. The interaction between SFs and TDs leads to the bending of TDs in the vicinity of SFs [Fig. 3(c)] or even to the termination of TDs directly on the SFs. ${ }^{41}$ The voids are elongated in the growth direction. Their vertical size varies between 50 and $750 \mathrm{~nm}$, while their lateral width ranges between 20 and $200 \mathrm{~nm}$. The highest density of the voids was found approximately in the middle of the structured layer.

In the top layer, the density of TDs is significantly reduced [Fig. 3(c)]. Mainly the TDs, which are located near the boundaries of the vertical columns in the structured layer, are transmitted into the uppermost GaN layer. These TDs have a form of dislocation bundles. In contrast to the interface between the template layer and the structured layer, the interface between the structured layer and the top layer is smooth. If the dislocation bundles in the top GaN layer would be treated as single TDs, their density in the top layer would be about $10^{8} \mathrm{~cm}^{-2}$ as calculated for the lateral size of the columns of approximately $1 \mu \mathrm{m}$. In reality, the (mean) dislocation density is higher because of the high TD density in dislocation bunches.

\section{B. Depth profile of the threading dislocation density}

The changes in the TD density along the GaN stack (sample S1) were quantified with the aid of nanofocused synchrotron diffraction. Typical two-dimensional reciprocal space maps (RSMs) $10 \overline{1} 0$ recorded for the template layer, for the structured layer, and for the top GaN layer are displayed in Fig. 4. The template layer

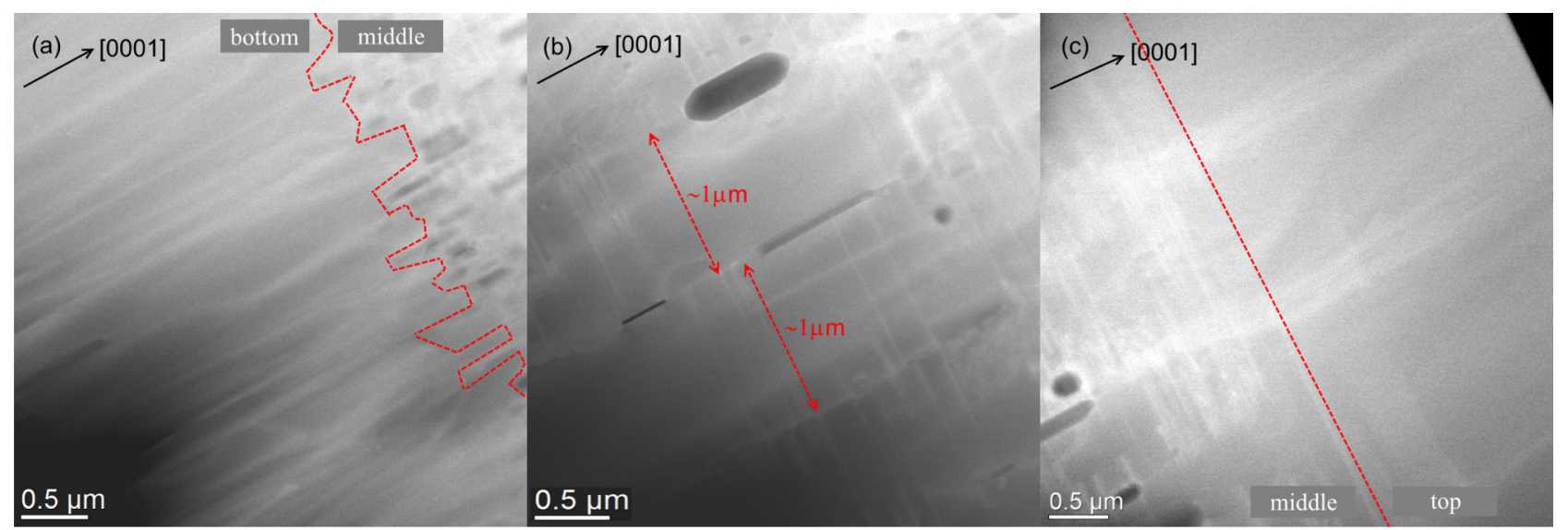

FIG. 3. Dark-field STEM images of the GaN template (a), structured GaN layer (b), and top GaN layer (c). The dashed line in panel (a) shows the jagged interface between the GaN template and the structured layer. The arrows in panel (b) mark two columnar grains terminated by dislocation walls. The dashed line in panel (c) highlights the interface between the structured layer and the top layer. 

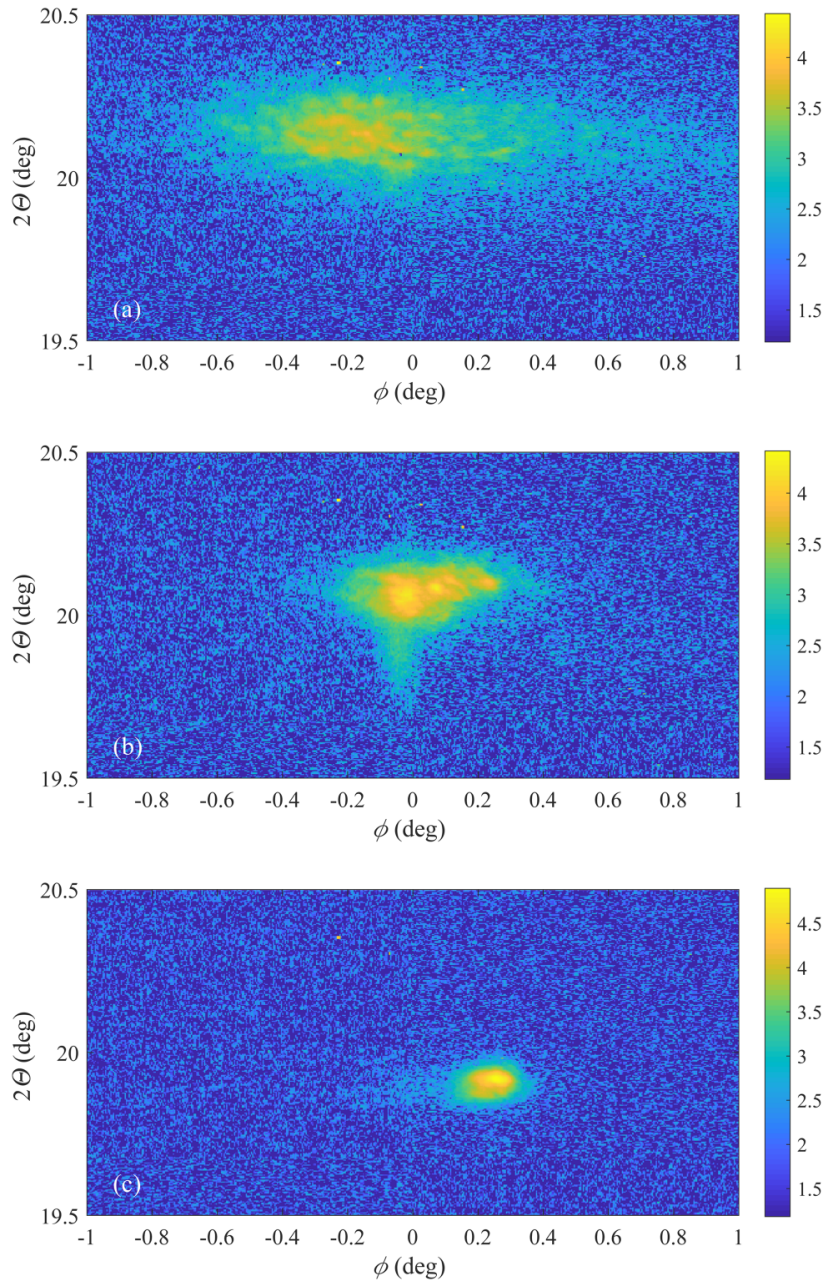

FIG. 4. Examples of the $10 \overline{10} \mathrm{GaN}$ reciprocal space maps measured in the template layer (a), at the interface between the template and the structured layer (b), and in the top GaN layer (c). The intensities (total counts) are plotted in a logarithmic scale. The orientation of the $2 \mathrm{D}$ detector is the same as in Fig. 2.

produces diffraction maxima that are strongly broadened in the radial $(2 \theta)$ and azimuthal $(\phi)$ directions, cf. Fig. 4(a). The $10 \overline{1} 0$ GaN RSM measured at the interface between the template and the structured layer [Fig. 4(b)] displays two diffraction maxima that are located at different $2 \theta$ angles. The strong intensity maximum occurring at larger $2 \theta$ resembles the RSM from the template. However, it is narrower in the $\phi$ direction than the diffraction maximum from the template [Fig. 4(a)]. Within the structured layer, the diffraction maximum from the template becomes successively narrower in the $\phi$ direction and weaker, because it is continually replaced by the diffraction spot located at a smaller $2 \theta$ angle. The new intensity maximum is narrow in the $\phi$ direction and broad in the $2 \theta$ direction [Fig. 4(b)]. It seems that the first part of the structured layer contains two kinds of GaN crystallites. The first one is similar to the crystallites in the template, while the second one is different with respect to the lattice parameter and the TD density. In the second part of the structured layer, only the second kind of crystallites (the new ones) grow. The top GaN layer produces a single diffraction maximum, which is located at a smaller $2 \theta$ angle and, in contrast to the previous layers, narrow in the $2 \theta$ and $\phi$ directions [Fig. 4(c)].

In the sample orientation from Fig. 2, the edge TDs with the Burgers vectors ${ }^{6} \vec{b}_{\text {edge }}^{(1)}=[110] a / 3$ and $\vec{b}_{\text {edge }}^{(2)}=[100] a / 3$ produce twist of the mosaic blocks, ${ }^{50}$ which broadens the diffraction maxima $10 \overline{1} 0$ in the $2 \theta$ direction (cf. Fig. 4). For this diffraction line, the angle between the diffraction vector and the respective Burgers vector, $\vec{b}_{\text {edge }}^{(1)}$ or $\vec{b}_{\text {edge }}^{(2)}$, is $30^{\circ}$ and $150^{\circ}$. The edge TDs with the Burgers vector $\vec{b}_{\text {edge }}^{(3)}=[0 \overline{1} 0] a / 3$ have no effect on the positions and shape of the $10 \overline{1} 0$ Bragg peak, because their Burgers vector is perpendicular to the diffraction vector (cf. Fig. 5). Analogously, the Burgers vectors of screw TDs $\vec{b}_{\text {screw }}^{\dagger}=[001] c$ and $\vec{b}_{\text {screw }}^{\downarrow}=[00 \overline{1}] c$ and the Burgers vector $[0 \overline{1} 3]$ of mixed $\mathrm{TDs}^{6}$ are perpendicular to the diffraction vector $(10 \overline{10})$, and thus invisible for diffraction. The contributions of crystallographically equivalent mixed TDs with Burgers vectors oriented along [113] and [103] to the diffraction phenomena are negligible, because these Burgers vectors make the angles of $80^{\circ}$ and $100^{\circ}$ with the diffraction vector (10 $\left.\overline{1} 0\right)$, as calculated for the lattice parameters of $\mathrm{GaN}, a=3.19 \AA$ and $c=5.19 \AA$.

According to the mosaicity model, the line broadening measured as a full width at half maximum in the $2 \theta$ direction $(\beta)$ can

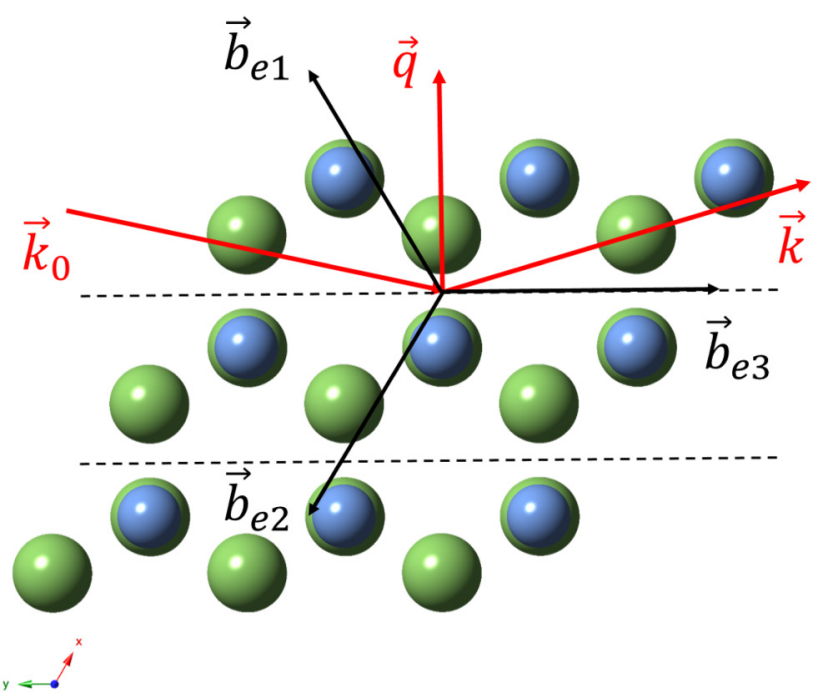

FIG. 5. Alternative orientations of the Burgers vectors of edge TDs with respect to the orientation of the diffraction vector $\vec{q} . \vec{k}_{0}$ is the wave vector of the primary beam, and $\vec{k}$ is the wave vector of the diffracted beam. The vectors $\vec{b}_{\text {edge }}^{(1)}=[110] a / 3, \vec{b}_{\text {edge }}^{(2)}=[100] a / 3, \vec{b}_{\text {edge }}^{(3)}=[0 \overline{10}] a / 3, \vec{q}=2 \pi(10 \overline{1} 0) / d_{1010}$, $\vec{k}_{0}$ and $\vec{k}$ lie in the same plane. This plane is perpendicular to the diffracting lattice planes (1010), which are plotted by dashed lines. In the definitions of the Burgers vectors and diffraction vector, $a$ is the lattice parameter of $\mathrm{GaN}$ and $d_{10 \overline{10}}$ is the interplanar spacing of the lattice planes $(10 \overline{10})$. 
be recalculated into the density of edge TDs using Eq. (2), ${ }^{4-6}$

$$
\rho_{e}=\frac{\beta^{2}}{4.35 \cdot b_{e}^{2}} \text {. }
$$

Equation (2) neglects the contribution of the small lateral crystallite size to the observed line broadening. At the mean lateral size of the $\mathrm{GaN}$ columns of $\approx 1 \mu \mathrm{m}$, the additional line broadening is about 10 arcsec and thus much smaller than the line broadening from dislocations ( $>250$ arcsec).

The dependence of the edge TD density on the distance from the sample surface is shown in Fig. 6(a). The template layer starts to grow with a density of edge TDs of approximately $2 \times 10^{9} \mathrm{~cm}^{-2}$, which is comparable with the edge TD density in GaN samples grown directly on sapphire substrates using other techniques, e.g.,
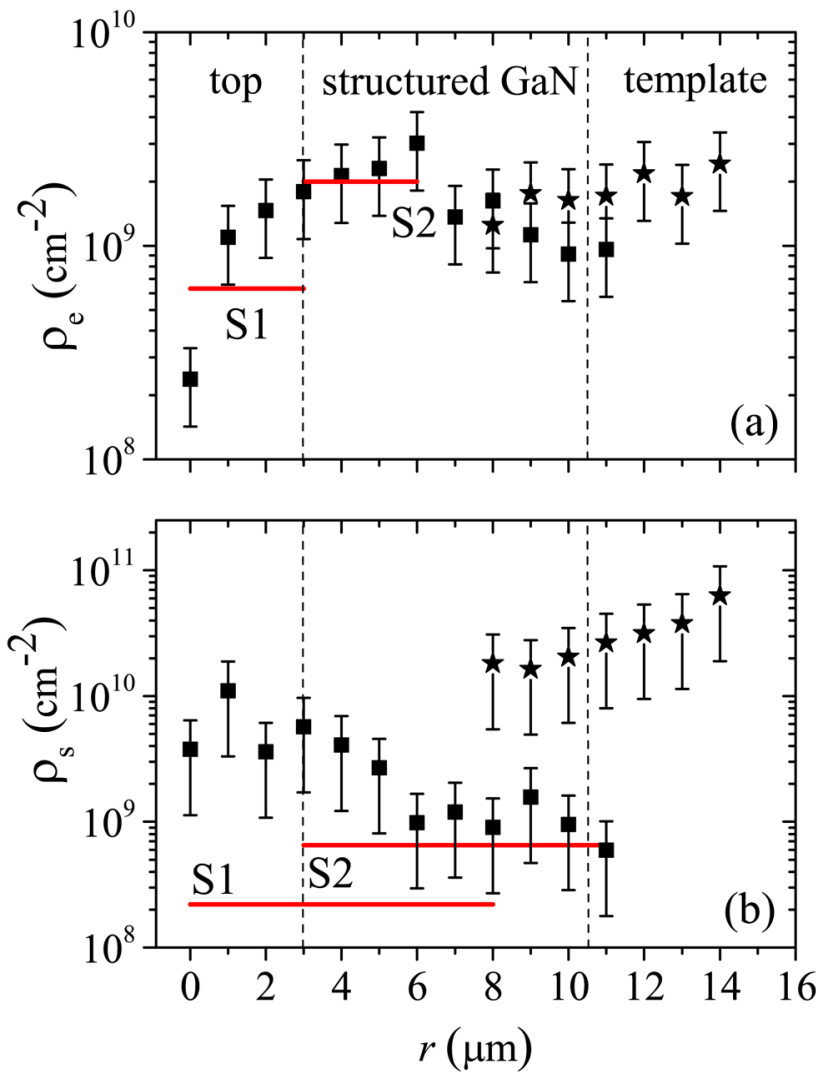

FIG. 6. Densities of edge (a) and screw TDs (b) calculated using the mosaicity model and plotted as a function of the distance from the sample surface. The asterisks denote the densities of TDs in the template and in grains growing from the template into the structured layer. The boxes stand for the TD densities in new grains of the structured layer and in the top GaN layer. Horizontal straight lines show the TD densities obtained from the Monte Carlo simulation of the reciprocal space maps $11 \overline{2} 4$ and 0004 that were measured in samples S1 and S2 using coplanar laboratory X-ray diffraction. The length of the straight lines gives the penetration depth of X-rays for the reflections $11 \overline{2} 4$ [panel (a)] and 0004 [panel (b)], and for the respective sample.
MOCVD. ${ }^{12}$ These TDs also survive in the first part of the structured layer. The new crystallites grow initially with a lower density of edge TDs. However, the density of edge TDs increases, as the "old" crystallites from the template disappear. A decrease of the edge TD density was first observed in the top GaN layer.

The densities of edge TDs obtained from the synchrotron diffraction experiment were verified by laboratory XRD measurements, which were carried out in reflection mode on samples S1 and S2. For laboratory XRD experiments, the GaN reflections 0004 and $11 \overline{2} 4$ were chosen (Fig. 7). The shape of the symmetrical reflection 0004 is only affected by screw TDs, and the shape of the asymmetrical reflection $11 \overline{2} 4$ depends on the density of both TD types. The dislocation densities were determined using a simulation routine based on the Monte Carlo (MC) method. ${ }^{12}$ Taking into account that the TD density obtained from the laboratory XRD
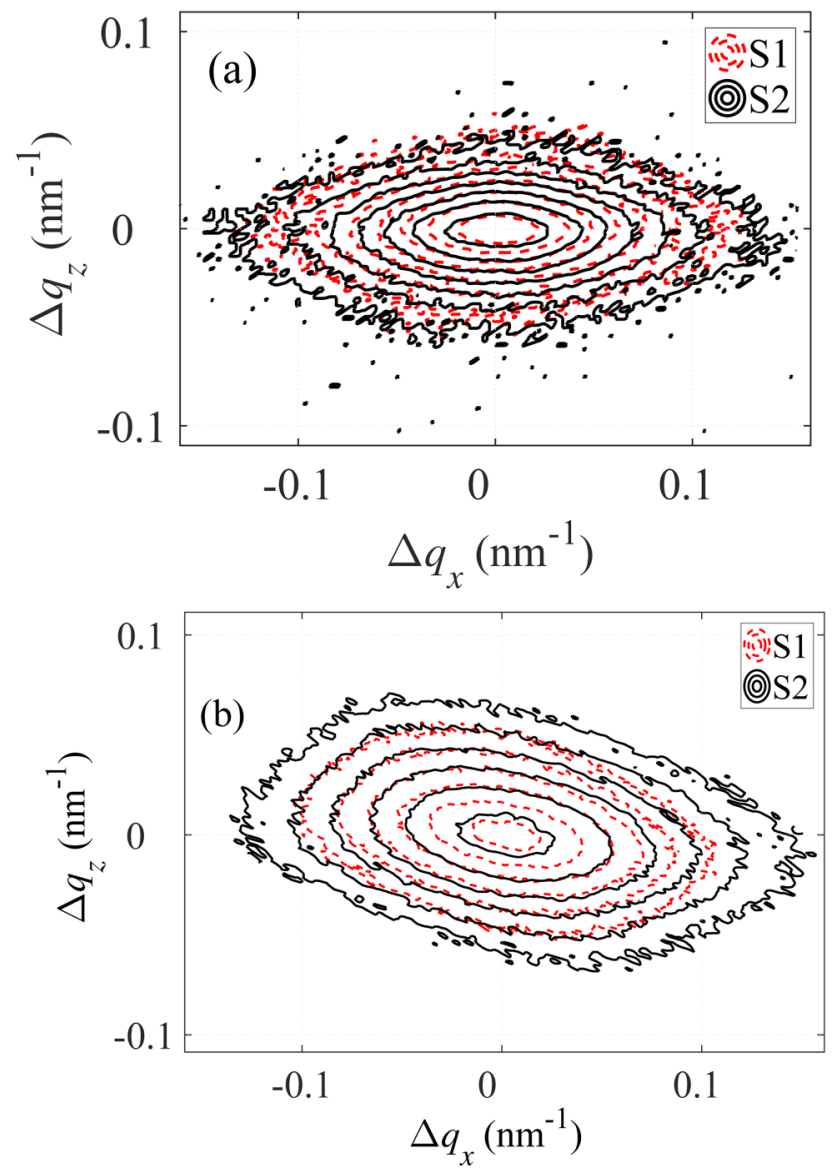

FIG. 7. Reciprocal space maps 0004 (a) and $11 \overline{2} 4$ (b) of $\mathrm{GaN}$ measured in the coplanar diffraction geometry. Dashed red contours belong to sample S1, and solid black contours correspond to sample S2. The intensity is plotted in a decimal logarithmic scale with an increment of $10^{0.3} . \Delta q_{x}$ and $\Delta q_{z}$ are the reciprocal space distances from the position of the respective intensity maximum. 
experiments [straight lines in Fig. 6(a)] is the mean value of the dislocation densities weighted by the reciprocal absorption coefficient, it can be concluded that the edge TD densities from the synchrotron experiment are in a very good agreement with the TD densities determined from the laboratory XRD data. The minimum density of edge TDs $\left(\approx 3 \times 10^{8} \mathrm{~cm}^{-2}\right)$ approaches the edge TD density reported for $\mathrm{GaN}$ grown by MOCVD on a $\mathrm{SiN}_{x}$ interlayer, ${ }^{12}$ on patterned sapphire substrates ${ }^{51}$ and on patterned sapphire substrates covered by an AlN nucleation layer, ${ }^{52}$ which are below $2 \times 10^{8} \mathrm{~cm}^{-2}$.

Screw TDs with the Burgers vectors $\vec{b}_{\text {screw }}^{\uparrow}=[001] c$ and $\vec{b}_{\text {screw }}^{\downarrow}=[00 \overline{1}] c$ are responsible for the tilt of the mosaic blocks. ${ }^{50}$ In the transmission diffraction geometry from Fig. 2, the tilt of the mosaic blocks broadens the diffraction spot 1010 in the azimuthal direction, i.e., along a "Debye ring" having the origin at $\vec{q}=0$ and the radius of $4 \pi \sin \theta_{B} / \lambda$, where $\theta_{B}$ is the Bragg angle of the reflection $10 \overline{1} 0$ and $\lambda$ is the wavelength of the synchrotron radiation. In contrast to edge TDs that contribute fully to the broadening in the radial direction (Fig. 4), the tilt of the mosaic blocks caused by screw TDs produces azimuthal line broadening, which depends on the tilt direction in this diffraction geometry.

If the growth direction of a mosaic block, which is the crystallographic direction [001] in our case, is tilted toward the diffraction vector, i.e., toward the vertical direction of the experimental setup (Fig. 2), the azimuthal shift of the respective diffraction spot is equal to the tilt of the corresponding block. Consequently, the mean tilt of the mosaic blocks is equal to the azimuthal line broadening. If a mosaic block is tilted around the diffraction vector, i.e., in the horizontal direction, the position of the diffraction spot does not change, which means that such tilt does not cause any line broadening in the diffraction setup used for this synchrotron experiment.

For these reasons, the density of screw TDs could not be determined unambiguously from the synchrotron diffraction experiment performed in the transmission mode on the reflection $10 \overline{1} 0$. The data plotted in Fig. 6(b) show rather the trend of the depth profile of the screw TD density than real screw TD densities. Reliable densities of screw TDs [horizontal lines in Fig. 6(b)] were obtained from the laboratory XRD experiment carried out on the diffraction line 0004 [Fig. 7(a)]. As mentioned above, the shape of this diffraction line is affected only by the screw TDs with the Burgers vectors $\vec{b}_{\text {screw }}= \pm[001] c$. A lower density of screw TDs in sample S1 than in sample S2 [Fig. 6(b)] indicates a slight reduction of the screw TD density in the top GaN layer that is analogous to the reduction of the edge TD density discussed above. The azimuthal broadening of the diffraction line $10 \overline{1} 0$ (Fig. 4) measured in the transmission geometry was used to characterize the development of the screw TD density during the layer growth. For conversion of the azimuthal line broadening $(\alpha)$ into the screw TD density, Eq. (3),

$$
\rho_{s}=\frac{\alpha^{2}}{4.35 \cdot b_{s}^{2}},
$$

was applied in analogy to Eq. (2). ${ }^{6}$ In the template and in the structured layer, the density of screw TDs shows a trend, which is similar to the density of edge TDs (cf. Fig. 6). The screw TD density is highest in the template and lowest in new crystallites growing at the beginning of the structured layer. In contrast to the density of edge TDs, the density of screw TDs is not reduced significantly in the top layer.

The effect of the structured layer on the reduction of the TD density in the GaN films grown directly on (0001)-oriented sapphire substrates can additionally be illustrated by the comparison of the TD densities in samples under study (grown using the threestep technique) and in $\mathrm{GaN}$ samples grown by a single-step HTVPE method. In GaN samples grown without the structured layer (using the single-step technique), ${ }^{42}$ the density of edge TDs was approximately 3 times higher than in the GaN samples grown using the three-step technique (see sample S1 in Fig. 6). The ratio of the screw TD densities in samples without and with the structured layer was about 4 . The TD densities in "nonstructured" samples $\quad\left(\rho_{e}=1.5-2 \times 10^{9} \mathrm{~cm}^{-2}, \quad \rho_{s}=0.5-1.1 \times 10^{9} \mathrm{~cm}^{-2}\right)^{42}$ approach the TD densities determined in sample S2 $\left(\rho_{e} \approx 2 \times 10^{9} \mathrm{~cm}^{-2}, \rho_{s} \approx 6 \times 10^{8} \mathrm{~cm}^{-2}\right.$, cf. Fig. 6). Lower TD densities were found in previous $\mathrm{GaN}$ samples containing similar voids like in the "structured" sample (cf. Fig. 3). The TD densities in these samples were $\rho_{e}=9 \times 10^{8} \mathrm{~cm}^{-2}$ and $\rho_{s}=2 \times 10^{8} \mathrm{~cm}^{-2}$. ${ }^{41}$

\section{Reliability of applied experimental techniques}

The TEM observations (Fig. 3) identified the dislocation bunching accompanied by dislocation annihilation, the interaction between TDs and SFs on basal lattice planes, and the deflection of TDs on voids present in the structured GaN layer as the prominent mechanisms of the TD density reduction. The voids mainly facilitate the dislocation bunching, while the SFs contribute to the dislocation bunching and partially terminate the dislocation propagation. Bunched TDs are typically localized near the walls of columnar GaN crystallites and are a typical phenomenon in the top GaN layer [Fig. 3(c)]. The bunching of TDs is known to modify the shape of the diffraction lines-it makes the diffraction lines Lorentz-shaped. ${ }^{16}$ The analysis of the shape of the rocking curves 0004, which were measured using laboratory XRD in the symmetrical reflection diffraction geometry, revealed that $30 \%$ of screw TDs in sample S2 are bunched, while the degree of the screw TDs bunching in sample S1 approached 50\%. The accumulation of the dislocation bunching was already confirmed by TEM (Fig. 3).

From the microstructure point of view, the SFs on basal lattice planes reduce the density of TDs in (0001)-oriented GaN. Concurrently, the presence of basal SFs increases the broadening of all diffraction lines in the $c^{*}$ direction of the reciprocal space, which also includes an additional azimuthal broadening of the diffraction line $10 \overline{1} 0$ measured in the transmission mode, and leads consequently to a systematic increase of the corresponding screw TD densities. Therefore, the densities of screw TDs in Fig. 6(b) that were determined from the azimuthal broadening of the diffraction line $10 \overline{1} 0$ are systematically higher than the screw TD densities obtained from the laboratory experiment done in the reflection mode.

Another microstructure feature that broadens the Bragg peaks is a limited lateral size of the columnar GaN crystallites. ${ }^{50}$ In this particular case, the diffraction lines are broadened in the $a^{*}$ and $b^{*}$ directions of the reciprocal space. Thus, the presence of the boundaries between neighboring mosaic blocks may apparently increase 
the density of edge TDs determined from radial $(2 \theta)$ broadening of the diffraction line $10 \overline{10}$ measured in the transmission diffraction geometry. However, this effect is much weaker than the effect of the SFs, as it can be seen from a good agreement of the densities of edge TDs that were determined using laboratory XRD and synchrotron diffraction [Fig. 6(a)].

When comparing the TD densities determined using the laboratory $\mathrm{XRD}$ in reflection mode and synchrotron diffraction in transmission mode, it is worth noting that these experimental methods are complementary regarding their sensitivity to the respective kind of TDs and other microstructure defects. While the $\mathrm{XRD}$ in reflection mode recognizes the tilt of the mosaic blocks predominantly in (0001)-oriented GaN crystals, the XRD in the transmission mode is sensitive to both, tilt and twist. The SFs on basal lattice planes do not affect the broadening of diffraction lines measured in the symmetrical reflection diffraction geometry, but broaden strongly the diffraction line $10 \overline{1} 0$ measured in the transmission geometry. Thus, the presence of the SFs increases apparently the density of screw TDs determined from the azimuthal broadening of the reflection $10 \overline{1} 0$. The limited lateral size of columnar mosaic blocks affects the density of screw TDs determined using XRD in reflection mode and the density of edge TDs determined using XRD in transmission mode, but this effect was relatively weak in the sample under study. Thus, the density of screw TDs can be obtained reliably using the reflection geometry, while the density of edge TDs follows directly from the line broadening measured in the transmission diffraction geometry. Additionally, the use of the nanobeam diffraction in the transmission mode made the quantification of the changes in the TD densities during the growth possible.

\section{Effect of the growth conditions on the lattice parameters}

The positive effect of the structured GaN layer on the dislocation bunching and consequently on the formation of wider columnar crystallites was already discussed above. An additional effect of the variation of the deposition parameters was indicated by the presence of two intensity maxima in Fig. 4(b), but not considered in detail yet. As the intensity maxima lie at different diffraction angles $(2 \theta)$, they refer to different in-plane lattice parameters $a$. The dependence of the lattice parameter $a$ on the distance from the sample surface is displayed in Fig. 8(a). The template layer possesses a lattice parameter $a$, which is smaller than the intrinsic lattice parameter $3.1894 \AA{ }^{53}$ New crystallites in the structured layer start to grow with a larger lattice parameter $a$. In the top GaN layer, the in-plane lattice parameter approaches the intrinsic value. The lattice parameter obtained from the nanobeam synchrotron diffraction experiments was verified by selected area electron diffraction (SAED) in TEM at several positions in the sample [Fig. 8(a)]. In order to increase the precision of the lattice parameters determined using SAED, the SAED patterns of $\mathrm{GaN}$ were calibrated with the aid of the SAED patterns from sapphire substrate.

Furthermore, the SAED patterns were used to determine the out-of-plane lattice parameter $c$ [Fig. 8(b)], which is not directly accessible in the synchrotron diffraction experiment performed on
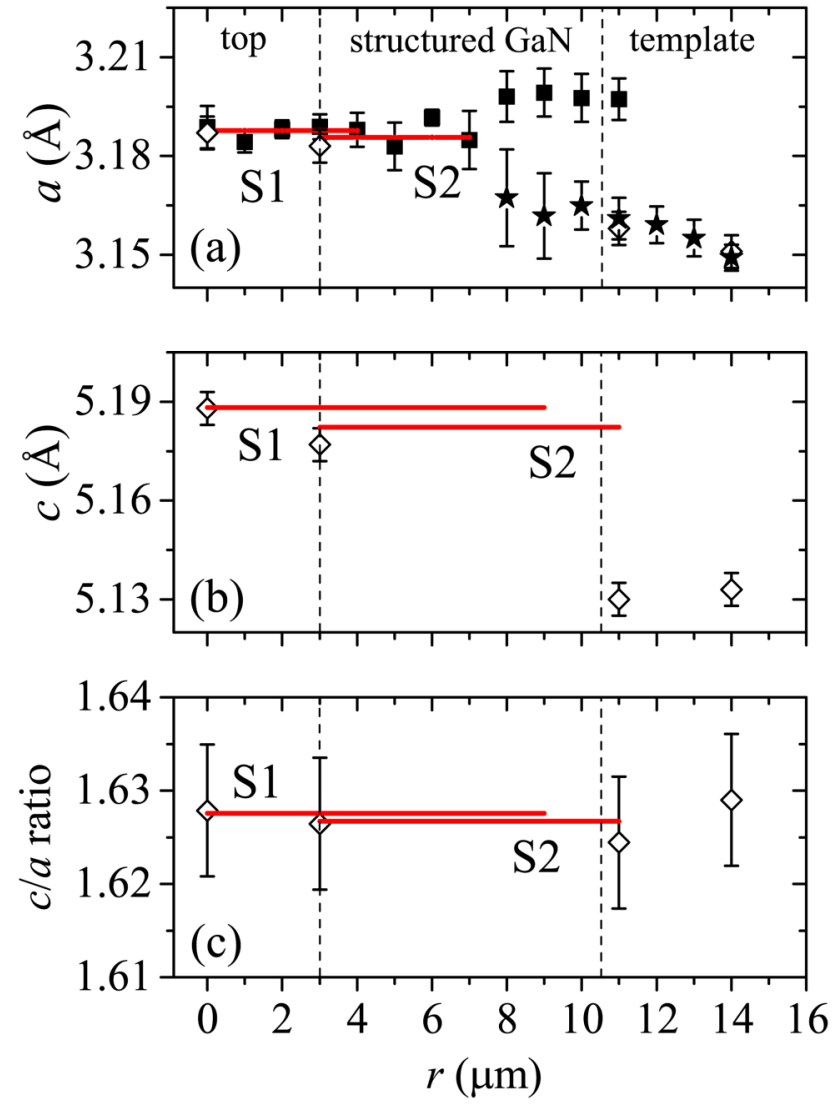

FIG. 8. Dependence of the GaN lattice parameters a (a) and $c(b)$ and the $c / a$ ratio (c) on the distance from the sample surface $(r)$. Individual lattice parameters $a$ and $c$ were calculated from the positions of the diffraction line 1010 (filled symbols) and from the SAED patterns (open symbols). The averaged lattice parameters (horizontal lines) were determined from the positions of the GaN reflections 0004 and $11 \overline{2} 4$. The length of the horizontal lines shows the penetration depth of $X$-rays for the respective reflection and for the respective sample. Vertical dashed lines mark the three zones of sample S1. The meaning of the filled symbols (asterisks and boxes) in panel (a) is the same like in Fig. 5.

the $10 \overline{10} \mathrm{GaN}$ Bragg diffraction. The lattice parameter $c$ shows a similar trend like the lattice parameter $a$. In the template, the lattice parameter $c$ starts far below the intrinsic value of $5.18614 \AA^{53}$ and achieves its intrinsic values in the top GaN layer as well. Consequently, the GaN stack starts to grow with a smaller volume of the elementary cell in the template. The intrinsic volume of the elementary cell is attained in the top layer. The $c / a$ ratio stays constant (within the experimental accuracy) throughout the whole stack, which indicates that the observed changes in the lattice parameters are not caused primarily by residual stresses. This result was supported by confocal micro-Raman spectroscopy, which revealed the almost constant shift of the spectral position of the $E_{2}$ (high) phonon over the whole sample S1 (Fig. 9). As the frequency of the $E_{2}$ (high) phonon is shifted to higher values as compared to the strain-free bulk $\mathrm{GaN},{ }^{45}$ the $\mathrm{GaN}$ stack $\mathrm{S} 1$ seems to be 


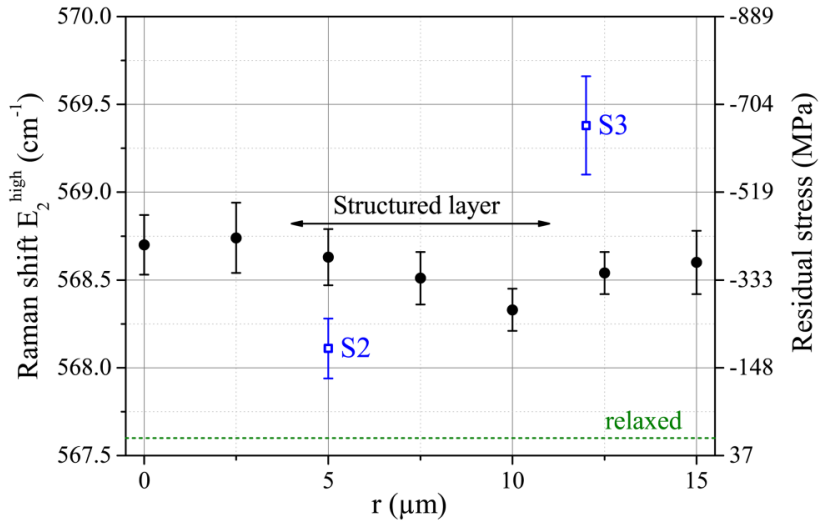

FIG. 9. Raman shift of the $E_{2}$ (high) Raman mode as a function of the distance from the surface of sample $\mathrm{S} 1$ (filled circles). Open boxes denote positions of the $E_{2}$ (high) Raman mode measured on the surface of samples S2 (ending with structured $\mathrm{GaN}$ ) and $\mathrm{S} 3$ (template). The green horizontal dashed line indicates the $E_{2}$ (high) frequency of strain-free bulk $\mathrm{GaN}$.

under compressive residual stress, which amount is, according to Eq. (1), about 300-500 MPa.

Higher residual stress was found at the upper end of the template (S3 in Fig. 9), a lower residual stress at the end of the structured GaN layer (S2 in Fig. 9). This trend can also be found in the depth-resolved measurement (filled circles in Fig. 9), which shows the minimum of the residual stress near the interface between the template and the structured layer that is followed by a slight increase of the residual stress in the top GaN layer. However, it must be taken into account that the differences between the individual residual stress values measured in sample S1 are smoothed through the mechanical interaction between individual parts of the $\mathrm{GaN}$ stack and/or through the convolution of the spectral positions of the $E_{2}$ (high) phonon in the confocal micro-Raman spectroscopy.

Complementary information about the changes of the lattice parameters during the growth of the GaN stack was obtained from the laboratory XRD measurements performed in reflection mode on samples S1 and S2 (Fig. 1), which revealed lattice parameters averaged over the penetration depth of the X-ray radiation (horizontal lines in Fig. 8). For both samples, the lattice parameters $a$ and $c$ were calculated from the $q_{x}$ and $q_{z}$ positions of the diffraction lines 0004 and $11 \overline{2} 4$ using a least-squares refinement algorithm. ${ }^{54}$ Also, these values confirm the increase of both lattice parameters toward the top GaN layer.

Reduced lattice parameters and small elementary cell volume in the template and in the structured GaN layer obviously result from the deposition conditions, in particular from the growth under a low V/III ratio. The nitrogen-deficient growth of GaN facilitates formation of structural vacancies in the nitrogen sublattice, which is assumed to be the main reason for the observed reduction of the lattice parameters and the elementary cell volume. The highest V/III ratio of about 5000 was established during the deposition of the top layer. These conditions led to the growth of $\mathrm{GaN}$ with "intrinsic" lattice parameters. The reduction of lattice parameters caused by the presence of the nitrogen vacancies affects possibly the results of the Raman spectroscopy. The shift of the Raman mode $E_{2}$ (high) is related to a change of phonon vibration frequency, which reflects any distortion of the elementary cell. Thus, the observed Raman shift cannot be unambiguously assigned to the residual stress, but it is a result of the lattice distortion produced by different kinds of the lattice strain.

\section{CONCLUSIONS}

Results of this study confirmed that the interaction of microstructure defects can be utilized to improve the quality of polar $\mathrm{GaN}$ crystals grown directly on (0001)-oriented sapphire substrates. In this particular case, the role of a structured GaN layer deposited with increased growth rate and at a lower substrate temperature was investigated. These deposition parameters primarily diminish the surface diffusivity of $\mathrm{GaN}$ during the growth, which leads to the formation of elongated voids between neighboring $\mathrm{GaN}$ crystallites. Together with the basal stacking faults in GaN, these voids facilitate the bunching and annihilation of unwanted threading dislocations that arise to compensate the lattice misfit at the GaN/sapphire interface. The reduction of the threading dislocation density was confirmed by transmission electron microscopy, nanofocused X-ray beam synchrotron diffraction carried out in the transmission mode and laboratory X-ray diffraction performed in the reflection mode. The combination of nanobeam synchrotron diffraction and selected area electron diffraction in the transmission electron microscope revealed the dependence of the lattice parameters on the distance from the substrate. A correlation of the depth profile of the microstructure parameters with the parameters of the deposition process have shown that a decrease of the V/III ratio leads to a reduction of the volume of the elementary cell of GaN. In this context, the importance of a straightforward modification of the deposition parameters in a single deposition process and the potential of the modified high-temperature vapor phase epitaxy for the defect engineering were illustrated.

\section{ACKNOWLEDGMENTS}

The authors thank the Deutsches Elektronen-Synchrotron (DESY) for the beam time at the end-station P 03 and A. Leuteritz for the preparation of the specimens for transmission electron microscopy. D.R. and O.P. acknowledge the financial support of Project No. 257632561 (PA 1236/3-3 and RA 1050/28-3) by the German Research Foundation (DFG). S.L. acknowledges Tomsk Polytechnic University Competitiveness Enhancement Program grant (Project No. VIU-MNOL NK-214/2018).

\section{REFERENCES}

${ }^{1}$ F. Medjdoub, Gallium Nitride (GaN): Physics, Devices, and Technology (CRC Press, 2017).

${ }^{2}$ D. Ehrentraut, E. Meissner, and M. Bockowski, Technology of Gallium Nitride Crystal Growth (Springer-Verlag, Berlin, Heidelberg, 2010).

${ }^{3}$ J. Elsner, R. Jones, P. K. Sitch, V. D. Porezag, M. Elstner, T. Frauenheim, M. I. Heggie, S. Öberg, and P. R. Briddon, Phys. Rev. Lett. 79, 3672-3675 (1997).

${ }^{4}$ P. Gay, P. Hirsch, and A. Kelly, Acta Metall. 1, 315-319 (1953).

${ }^{\mathbf{5}}$ C. O. Dunn and E. F. Koch, Acta Metall. 5, 548-554 (1957). 
${ }^{6}$ T. Metzger, R. Höpler, E. Born, O. Ambacher, M. Stutzmann, R. Stömmer, M. Schuster, H. Göbel, S. Christiansen, M. Albrecht, and H. P. Strunk, Philos. Mag. A 77, 1013-1025 (1998).

7J. S. Speck and S. J. Rosner, Phys. B: Condens. Matter 273-274, 24-32 (1999).

${ }^{8}$ T. Hino, S. Tomiya, T. Miyajima, K. Yanashima, S. Hashimoto, and M. Ikeda, Appl. Phys. Lett. 76, 3421 (2000).

${ }^{9}$ V. M. Kaganer, O. Brandt, A. Trampert, and K. H. Ploog, Phys. Rev. B 72, 1013 (2005).

${ }^{10}$ V. M. Kaganer, O. Brandt, H. Riechert, and K. K. Sabelfeld, Phys. Rev. B 80, 033306 (2009).

${ }^{11}$ V. Holý, T. Baumbach, D. Lübbert, L. Helfen, M. Ellyan, P. Mikulík, S. Keller, S. P. DenBaars, and J. Speck, Phys. Rev. B 77, 094102 (2008).

${ }^{12} \mathrm{M}$. Barchuk, V. Holý, B. Miljević, B. Krause, T. Baumbach, J. Hertkorn, and F. Scholz, J. Appl. Phys. 108, 43521 (2010).

${ }^{13}$ A. Sakai, H. Sunakawa, A. Kimura, and A. Usui, Appl. Phys. Lett. 76, 442-444 (2000).

${ }^{14}$ R. Datta and C. J. Humphreys, Phys. Status Solidi C 3, 1750-1753 (2006).

${ }^{15}$ X. Weng, J. D. Acord, A. Jain, E. C. Dickey, and J. M. Redwing, J. Electron. Mater. 36, 346-352 (2007).

${ }^{16}$ M. Barchuk, V. Holý, and D. Rafaja, J. Appl. Phys. 123, 161552 (2018).

${ }^{17}$ E. D. Bourret-Courchesne, K. M. Yu, M. Benamara, Z. Liliental-Weber, and J. Washburn, J. Electron. Mater. 30, 1417-1420 (2001).

${ }^{18}$ J. Hertkorn, F. Lipski, P. Brückner, T. Wunderer, S. B. Thapa, F. Scholz, A. Chuvilin, U. Kaiser, M. Beer, and J. Zweck, J. Cryst. Growth 310, 4867-4870 (2008).

${ }^{19} \mathrm{H}$. Ashraf, D. V. Sridhara Rao, D. Gogova, D. Siche, R. Fornari, C. J. Humphreys, and P. R. Hageman, J. Cryst. Growth 312, 595-600 (2010).

${ }^{20}$ B. Kuhn and F. Scholz, Phys. Status Solidi A 188, 629-633 (2001).

${ }^{21}$ J. Hertkorn, P. Brückner, S. B. Thapa, T. Wunderer, F. Scholz, M. Feneberg, K. Thonke, R. Sauer, M. Beer, and J. Zweck, J. Cryst. Growth 308, 30-36 (2007).

${ }^{22}$ J. Bläsing, A. Krost, J. Hertkorn, F. Scholz, L. Kirste, A. Chuvilin, and U. Kaiser, J. Appl. Phys. 105, 033504 (2009).

${ }^{23}$ H. V. Stanchu, A. V. Kuchuk, Y. I. Mazur, C. Li, P. M. Lytvyn, M. Schmidbauer, Y. Maidaniuk, M. Benamara, M. E. Ware, Z. M. Wang, and G. J. Salamo, Cryst. Growth Des. 19, 200-210 (2019).

${ }^{24}$ D.-S. Wuu, H.-W. Wu, S.-T. Chen, T.-Y. Tsai, X. Zheng, and R.-H. Horng, J. Cryst. Growth 311, 3063-3066 (2009).

${ }^{25} \mathrm{M}$. Ali, A. E. Romanov, S. Suihkonen, O. Svensk, S. Sintonen, M. Sopanen, H. Lipsanen, V. N. Nevedomsky, N. A. Bert, M. A. Odnoblyudov, and V. E. Bougrov, J. Cryst. Growth 344, 59-64 (2012).

${ }^{26}$ F. Réveret, Y. André, O. Gourmala, J. Leymarie, M. Mihailovic, D. Lagarde, E. Gil, D. Castelluci, and A. Trassoudaine, J. Cryst. Growth 421, 27-32 (2015).

${ }^{27}$ C. T. Shelton, I. Bryan, E. A. Paisley, E. Sachet, J. F. Ihlefeld, N. Lavrik, R. Collazo, Z. Sitar, and J.-P. Maria, APL Mater. 5, 96109 (2017).

${ }^{28}$ J. Hartmann, F. Steib, H. Zhou, J. Ledig, L. Nicolai, S. Fündling, T. Schimpke, A. Avramescu, T. Varghese, A. Trampert, M. Straßburg, H.-J. Lugauer, H.-H. Wehmann, and A. Waag, J. Cryst. Growth 476, 90-98 (2017).

${ }^{29}$ K. Hiramatsu, K. Nishiyama, A. Motogaito, H. Miyake, Y. Iyechika, and T. Maeda, Phys. Status Solidi A 176, 535-543 (1999).
${ }^{30}$ K. Hiramatsu, J. Phys.: Condens. Matter. 13, 6961-6975 (2001).

${ }^{31}$ C. H. Chiu, H. H. Yen, C. L. Chao, Z. Y. Li, P. Yu, H. C. Kuo, T. C. Lu, S. C. Wang, K. M. Lau, and S. J. Cheng, Appl. Phys. Lett. 93, 81108 (2008).

${ }^{32}$ H. Geng, H. Sunakawa, N. Sumi, K. Yamamoto, A. Atsushi Yamaguchi, and A. Usui, J. Cryst. Growth 350, 44-49 (2012).

${ }^{33}$ M. Xiao, J. Zhang, X. Duan, H. Shan, T. Yu, J. Ning, and Y. Hao, Sci. Rep. 6, $23842(2016)$

${ }^{34}$ S. Bohyama, K. Yoshikawa, H. Naoi, H. Miyake, K. Hiramatsu, and T. Maeda, Phys. Status Solidi C 0, 2159-2162 (2003).

${ }^{35}$ T. Matsubara, S. Goubara, K. Yukizane, R. Inomoto, N. Okada, and K. Tadatomo, Phys. Status Solidi B 254, 1600716 (2017).

${ }^{36} \mathrm{C}$. He, W. Zhao, K. Zhang, L. He, H. Wu, N. Liu, S. Zhang, X. Liu, and Z. Chen, ACS Appl. Mater. Interfaces 9, 43386-43392 (2017).

${ }^{37}$ G. Lukin, C. Röder, M. Barchuk, G. Schreiber, O. Pätzold, J. Kortus, D. Rafaja, and M. Stelter, Phys. Status Solidi C 11, 491-494 (2014).

${ }^{38}$ T. Schneider, G. Lukin, F. Zimmermann, M. Barchuk, E. Niederschlag, O. Pätzold, and M. Stelter, J. Cryst. Growth 468, 212-215 (2017).

${ }^{39} \mathrm{G}$. Lukin, T. Schneider, M. Barchuk, F. Zimmermann, E. Niederschlag, O. Pätzold, and M. Stelter, Phys. Status Solidi A 214, 1600753 (2017).

${ }^{40}$ M. Barchuk, M. Motylenko, G. Lukin, C. Röder, O. Pätzold, and D. Rafaja, Cryst. Res. Technol. 53, 1700113 (2018).

${ }^{41}$ M. Barchuk, G. Lukin, F. Zimmermann, C. Röder, M. Motylenko, O. Pätzold, J. Heitmann, J. Kortus, and D. Rafaja, J. Electron. Mater. 46, 1612-1619 (2017).

${ }^{42}$ M. Barchuk, M. Motylenko, G. Lukin, O. Pätzold, and D. Rafaja, J. Appl. Cryst. 50, 555-560 (2017).

${ }^{43}$ R. Gröger, L. Leconte, and A. Ostapovets, Comput. Mater. Sci. 99, 195-202 (2015).

${ }^{44}$ C. Röder, F. Lipski, F. Habel, G. Leibiger, M. Abendroth, C. Himcinschi, and J. Kortus, J. Phys. D: Appl. Phys. 46, 285302 (2013).

${ }^{45}$ V. Y. Davydov, N. S. Averkiev, I. N. Goncharuk, D. K. Nelson, I. P. Nikitina, A. S. Polkovnikov, A. N. Smirnov, M. A. Jacobson, and O. K. Semchinova, J. Appl. Phys. 82, 5097-5102 (1997).

${ }^{46}$ M. Barchuk, C. Röder, Y. Shashev, G. Lukin, M. Motylenko, J. Kortus, O. Pätzold, and D. Rafaja, J. Cryst. Growth 386, 1-8 (2014).

${ }^{47}$ C. Krywka, J. Keckes, S. Storm, A. Buffet, S. V. Roth, R. Döhrmann, and M. Müller, J. Phys.: Conf. Ser. 425, 72021 (2013).

${ }^{48}$ C. Krywka, H. Neubauer, M. Priebe, T. Salditt, J. Keckes, A. Buffet, S. V. Roth, R. Döhrmann, and M. Mueller, J. Appl. Cryst. 45, 85-92 (2012).

${ }^{49}$ D. C. Creagh and J. H. Hubbell, International Tables for Crystallography Volume C: Mathematical, Physical and Chemical Tables (Springer, Dordrecht, 2006), Section 4.2.4, pp. 220-229.

${ }^{50}$ R. Chierchia, T. Böttcher, H. Heinke, S. Einfeldt, S. Figge, and D. Hommel, J. Appl. Phys. 93, 8918-8925 (2003).

${ }^{51}$ N. Okada and K. Tadatomo, Semicond. Sci. Technol. 27, 024003 (2012).

${ }^{52}$ S. Zhou, H. Hu, X. Liu, M. Liu, X. Ding, C. Gui, S. Liu, and L. J. Guo, Jpn. J. Appl. Phys. 56, 111001 (2017).

${ }^{53}$ W. Paszkowicz, S. Podsiadlo, and R. Minikayev, J. Alloys Compd. 382, 100-106 (2004).

${ }^{54}$ G. A. Novak and A. A. Colville, Amer. Mineral. 74, 488-490 (1989). 